\title{
A STUDY BY AN OPEN CIRCUIT METHOD OF THE INTRAPULMONARY MIXTURE OF INSPIRED AIR
}

\author{
BY \\ MARGARET R. BECKLAKE \\ From the Department of Medicine, Postgraduate Medical School of London, and the \\ Department of Medicine, University of the Witwatersrand, South Africa
}

(RECEIVED FOR PUBLICATION SEPTEMBER 13, 1951)

Of the various pulmonary function tests used by the clinician in the assessment of such conditions as emphysema, the study of inequality of distribution of inspired air to the lung spaces has only been developed recently. Several techniques have been described whereby disturbances of this sort may be detected (Birath, 1944 ; Bates and Christie, 1950 ; Robertson, Siri, and Jones, 1950 ; Bateman, 1950). In their work on the development of an open circuit method of measuring functional residual air Cournand, Baldwin, Darling, and Richards (1941) had noticed that the percentage of alveolar nitrogen after seven minutes' pure oxygen breathing tended to be higher in emphysematous subjects than in normal controls. This suggested to them that tidal air was less effective in washing out the lung spaces of emphysematous subjects, and furthermore that in the emphysematous subject the tidal air was probably unevenly distributed to the lung spaces. Consequently they adapted their open circuit method for measuring functional residual air (F.R.A.) in such a way that it would assess this lack of evenness of intrapulmonary distribution of inspired air.

By collecting expired air in a series of experiments, each of a different duration, Darling, Cournand, and Richards (1944) were able to follow the rate of nitrogen elimination from the lungs during oxygen breathing. From the amount of nitrogen washed out of the lungs, an estimate was made of the percentage nitrogen in the air remaining in the lung. These results were plotted against the number of breaths, and compared with a predicted curve. The latter was calculated from a formula which took into account the tidal air, pulmonary dead space, and functional residual air of the subject, and assumed that with each breath a volume of air equal to the tidal air minus the pulmonary dead space (effective tidal air) was distributed evenly throughout the lung spaces. The principal disadvantage of this method was that a mixing study of five points required six experiments (with about half-an-hour's interval between each to allow re-aeration of the lungs and tissues) so that the total period over which the patient was studied amounted to about three hours. During so long a period fortuitous changes in tidal air and respiratory level might invalidate comparisons between different experiments. The aim of the present study was to develop a method in which the relevant information could be obtained in a single experiment. 


\section{The Apparatus}

The apparatus is illustrated in Fig. 1. The patient inhaled oxygen from a Tissot spirometer through a box-valve, which directed his expired air through a six-way tap into one of a series of Douglas bags. The six-way tap consisted of two brass plates held in close apposition by a spring. The upper plate was pierced by a single brass tube, which was attached to the expiratory side of the box-valve. The lower plate was pierced by six such brass tubes, each with a Douglas bag attached. The bottom plate could be rotated on the top plate so that each Douglas bag could be moved in turn into the position occupied by the left-hand bag in Fig. 1, and thus receive expired air. A stop mechanism

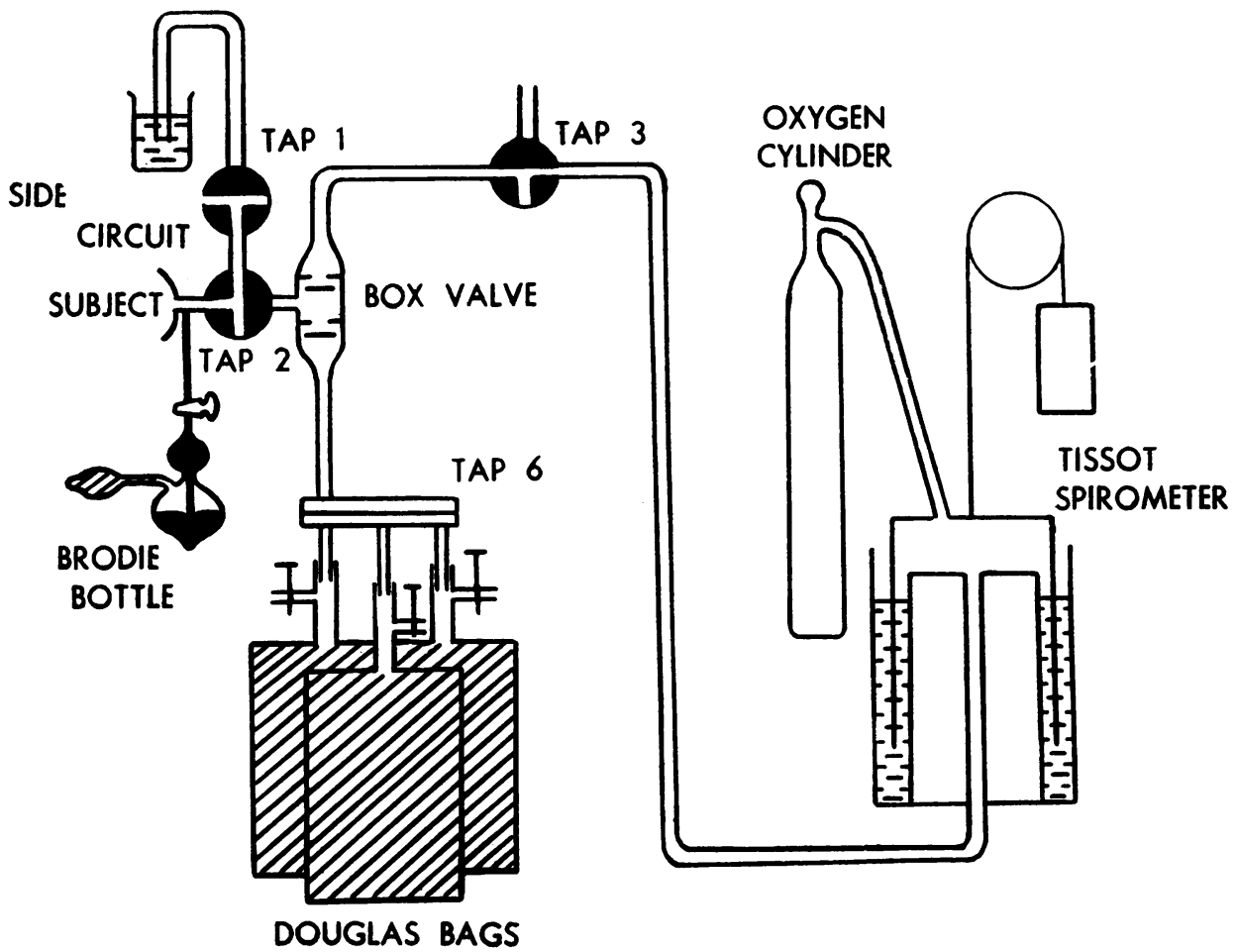

FIG. 1.-Schematic representation of the apparatus. The six-way tap $(\operatorname{tap} 6)$ is represented schematically in the diagram so that only three Douglas bags are visible.

ensured that the next bag in series was brought into a position exactly opposite the connecting tube each time the lower plate of the six-way tap was rotated. In this way, once oxygen breathing was begun, all the expired air could be collected serially into five separate Douglas bags.

Between the patient's mouthpiece and the box-valve was a side-circuit consisting of a tap, which could be opened to the atmosphere, or switched to a long glass tube closed by a water-seal. The purpose of the side-circuit was first to allow the subject to breathe atmospheric air before being switched on to breathing pure oxygen, and secondly, to allow the collection of an alveolar sample at the end of the mixing run, using the method described by Gilson and Hugh-Jones (1949). 


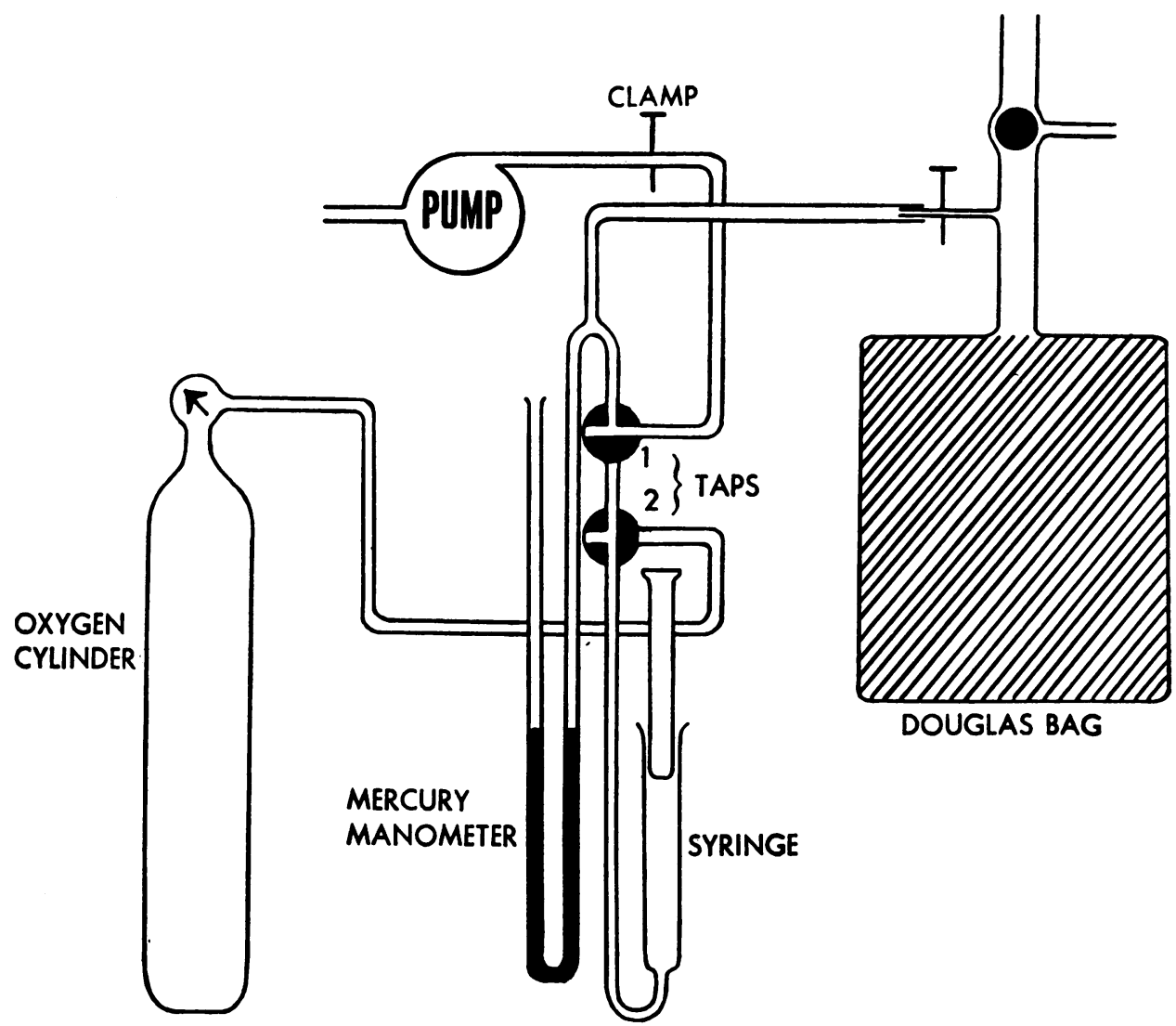

FIG. 2.-Diagram of the apparatus used for emptying the Douglas bags before a mixing run. The Douglas bag is emptied by means of a suction pump through its sampling tube, taps 1 and 2 turned as in figure. The rate of emptying is controlled by the clamp fixed on the tube connecting tap 1 to the pump. As soon as the bag is emptied and the mercury in the manometer begins to fall, tap 1 is switched to exclude the pump from the circuit, and oxygen is discharged from the loaded syringe back into the bag. The syringe can be re-loaded by turning tap 2 to connect it to the oxygen cylinder.

\section{Precautions}

Freeing the Apparatus of Nitrogen before a Mixing Run.-Care was necessary to ensure that the whole apparatus was flushed free of nitrogen before a mixing run, as a small amount of the latter gas, particularly in the last bags where the nitrogen content was usually low (1-2\%), would significantly disturb the results. Flushing out the Tissot spirometer presented no special problems as this was never used for anything except oxygen storage. Similarly the tubes connecting the Tissot spirometer to the box-valve were easily flushed with oxygen. The bags were flushed with oxygen and emptied by a suction pump attached to their sampling tubes (Fig. 2). A mercury manometer in a sidearm of the tube connecting the pump to the Douglas bag recorded the pressure at which the bag was emptied, usually $-5 \mathrm{~mm}$. $\mathrm{Hg}$. As soon as the pressure started to fall further the bag was considered empty and the pump switched out of the circuit. If the 
empty bags were allowed to stand before the mixing run, air began to leak back into them, so that it was necessary to add a known volume of pure oxygen to each bag immediately after it had been emptied, to abolish its slight negative pressure. This was done by discharging oxygen from a $100 \mathrm{cc}$. syringe, which had been incorporated into the apparatus for emptying bags (see Fig. 2), and which could be filled from the oxygen cylinder. Thus the apparatus could be freed of nitrogen before a mixing study and left standing up to a period of 30 minutes without any significant inward leak of atmospheric nitrogen.

Sampling from Douglas Bags. - Care was also necessary in sampling from the Douglas bags. To obtain a representative sample it is first necessary to wash out the side tube of the Douglas bag with an adequate volume of its contents. The expired air thus lost must be measured. Fig. 3 shows the apparatus used for sampling. Two bottles were arranged to aspirate a litre of air from the Douglas bag. Subsequently the connexion to the aspirating bottle was clamped, and a sample of the contents of the bag was collected in the Brodie bottle.

Measurement of the Volume of Gas in Each Douglas Bag.Careful trial showed that the usual method of expressing the contents of a Douglas bag

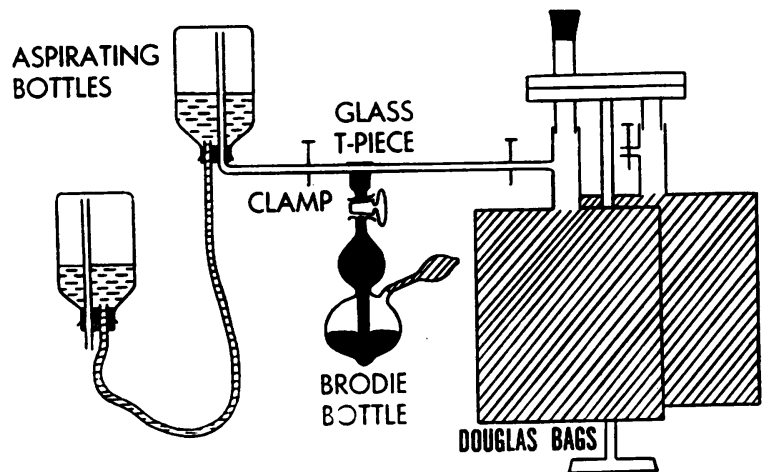

Fig. 3.-Diagram to show apparatus used for sampling from the Douglas bags.

through a gas meter was liable to introduce inaccuracies. Greater accuracy could be ootained when the bags were evacuated by a suction pump through a gas meter as shown in Fig. 4. It was essential that the tubing connecting the Douglas bag

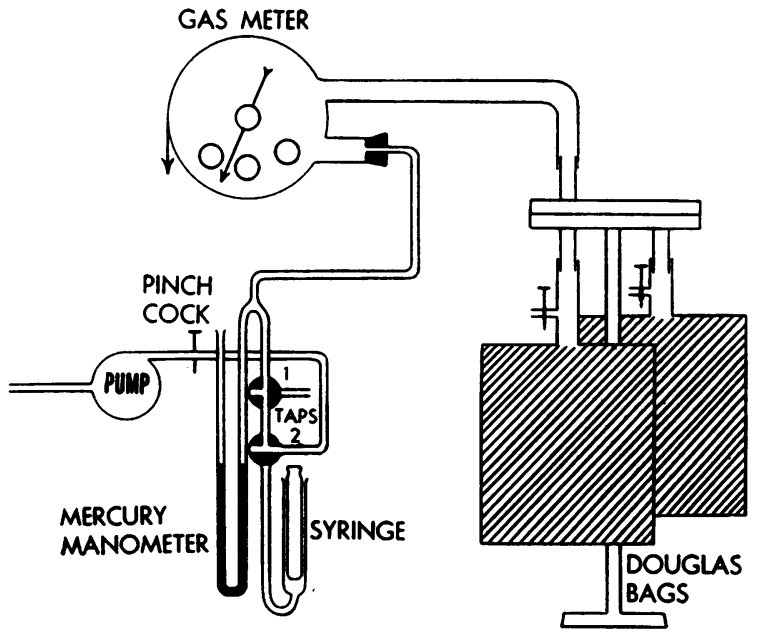

Fig. 4.-Diagram of apparatus used to measure the volumes of expired air collected in each Douglas bag. to the gas meter should be of wide bore, and also that the speed of the flow, regulated by a pinch-cock as shown in Fig. 4, be slow so that no significant negative pressure might develop in the circuit before the point at which the gas volume was measured. As soon as a bag was emptied, the pressure in the circuit began to fall. The pump was then switched out of circuit and the volume on the gas meter recorded. When a known volume of air was introduced into a Douglas bag and the bag evacuated in the manner described, the volume of air recovered agreed closely with the volume of air introduced. 


\section{Procedure}

The patient was connected to the apparatus, a nose-clip was fitted, and he breathed air through the side-arm of the apparatus with the taps turned as in Fig. 1. He was then instructed in the method of giving an alveolar sample (Gilson and Hugh-Jones, 1949) and practised this several times. Then tap 2 was turned to connect the patient to the oxygen supply and simultaneously, by rotating the six-way tap, the first bag was switched into a position to receive expired air. Ordinarily four breaths were collected into each of the first two bags, eight breaths into the third bag, 16 into the fourth bag, and 32 into the fifth bag. If the breathing was shallow larger numbers of breaths were collected in each bag. Oxygen breathing was continued for seven minutes whenever possible. At the end of the period of oxygen breathing, and at the height of an inspiration, the subject was switched to the side-circuit and an alveolar sample was collected. This completed the mixing run. The operator then sampled the gas mixture of each Douglas bag, taking two samples from the last bag as an additional check, and the volumes of gas in each Douglas bag were measured. The samples were analysed for nitrogen on a Haldane gas analysis apparatus by the dilution method (Peters and Van Slyke, 1932).

\section{EXPRESSION OF RESULTS}

In this study the degree of intrapulmonary mixing has been expressed as the " apparent functional residual air" (apparent F.R.A.), that is, that volume of lung which, if completely cleared of nitrogen, would yield the amount of nitrogen recovered in the expired air after any particular number of breaths (Briscoe, Becklake, and Rose, 1951). When all the nitrogen has been washed out of the subject's lungs, then the apparent F.R.A. is equal to the true F.R.A.

Mixing data were plotted with the apparent F.R.A. on a linear scale as ordinate against the number of breaths on a $\log _{2}$ scale as abscissa (Fig. 6). Assuming that each breath mixed evenly with the whole lung content, the apparent F.R.A.
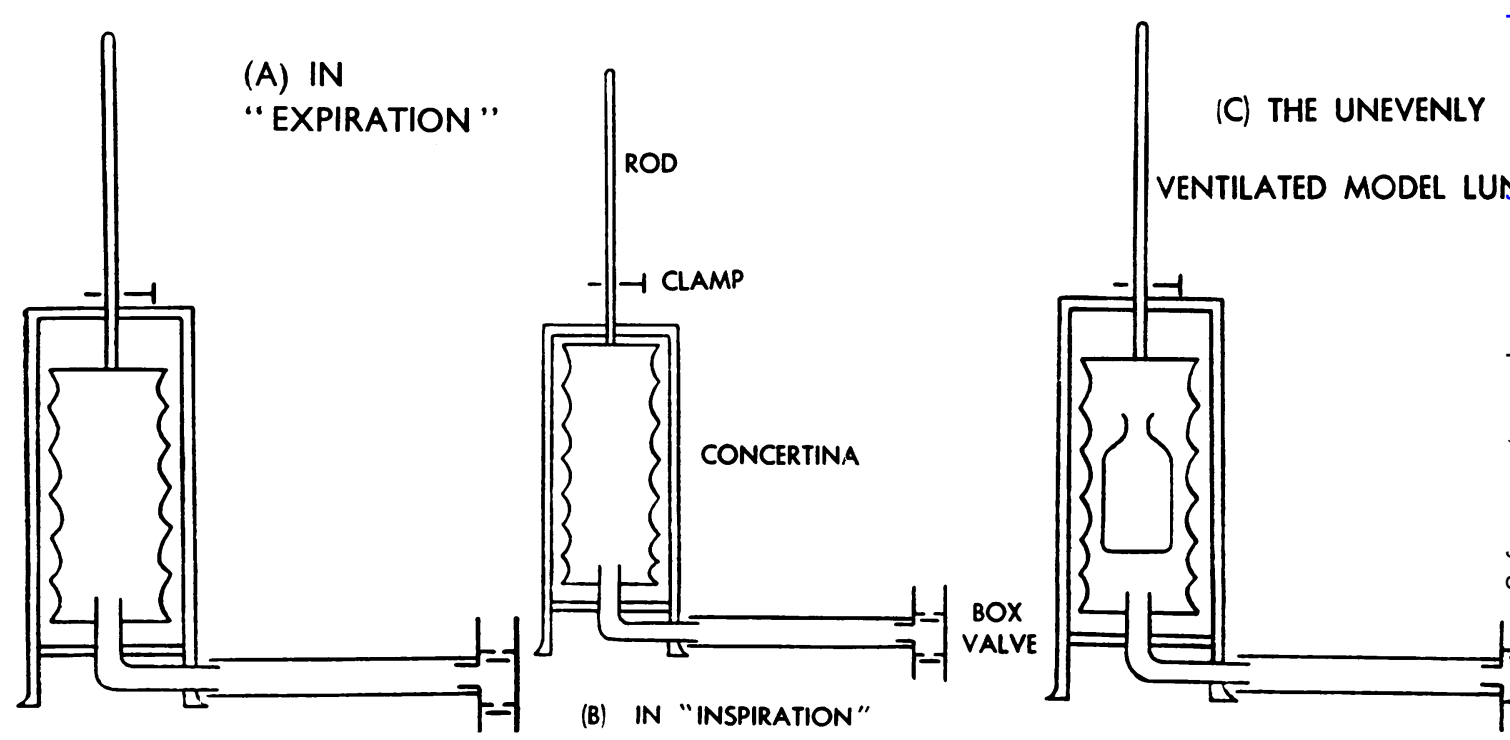

Fig. 5.-Diagram of the model lung.

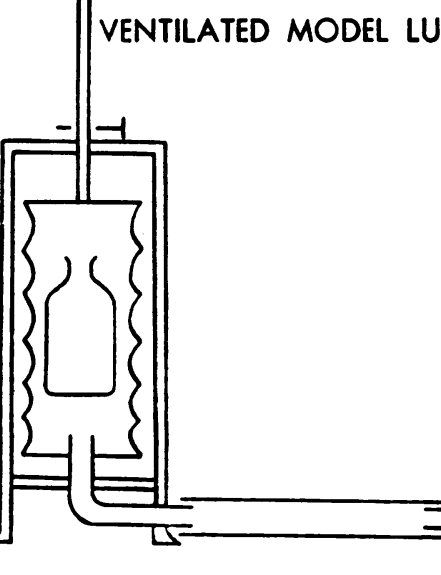


after any number of breaths could be predicted (see Appendix). For each subject several theoretical mixing curves were predicted and drawn. In Fig. 6, which shows the mixing data of a typical normal subject, the continuous lines are curves predicted for effective tidal airs (tidal air minus dead space) of $0.4,0.2$, and 0.1 litre. The

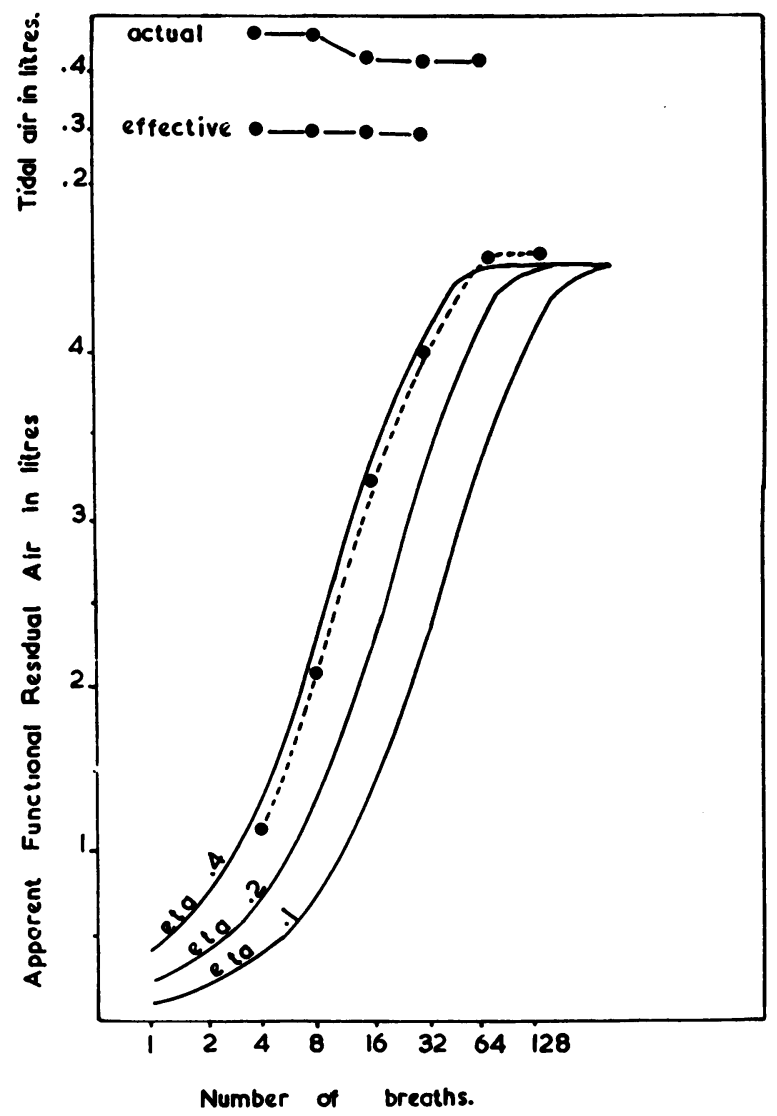

FIG. 6.-Mixing curve on a normal subject.

dotted line connects the experimental points obtained in this subject and lies between the 0.4 and 0.2 litre predicted curves. The effective tidal air read from the curve is plotted, with the actual tidal air, at the top of the diagram.

\section{Experiments to CheCK the Accuracy of the Method}

Experiments were done on a model lung to test the technique. The rubber concertina (Fig. 5) was ventilated by manual movement of the rod, the range of excursion being controlled by a clamp on the rod. The model is such that any increase in its tidal air will result in a corresponding decrease in its functional residual air ; in experiments where these two volumes vary, their sum should be constant. In three experiments, in which the functional residual air of the model 
was measured by the method described above, the total volumes (i.e., tidal air plus functional residual air) were $4.89,4.79$, and 4.91 litres respectively. The agreement between these figures demonstrates reasonable accuracy of the method.

In another experiment the model was filled with oxygen in the expiratory position, a litre of air was introduced by one inspiration, a sample of the resulting gas mixture was analysed, and the F.R.A. was estimated from the observed concentration of nitrogen. The total volume of the model was 4.85 litres, which agrees satisfactorily with the other estimates.

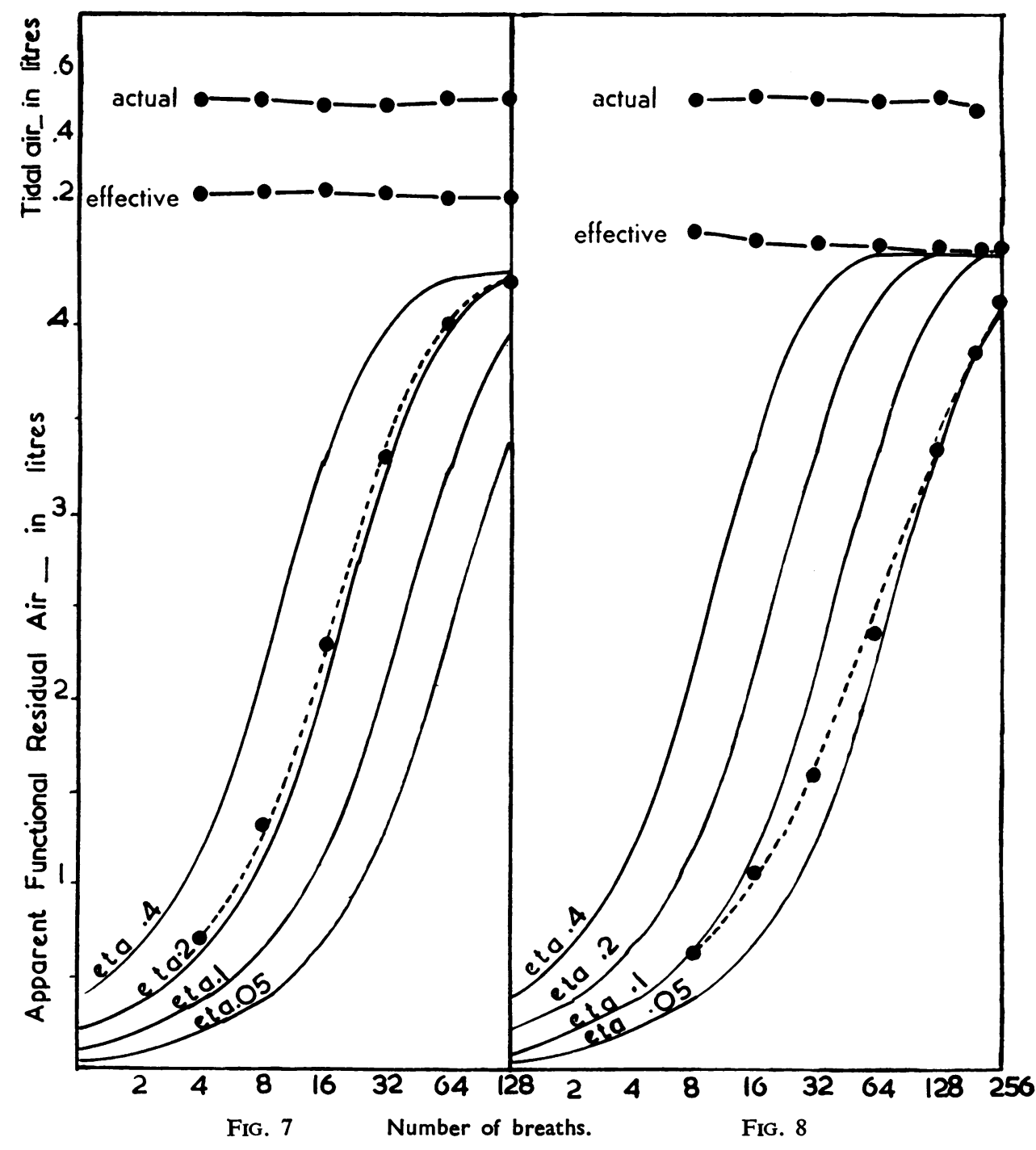

Figs. 7 and 8.-Mixing curves of the evenly (Fig. 7) and unevenly ventilated (Fig. 8) model lungs. 
It will be seen from Fig. 7 that the mixing curve of the model, calculated from one of the three experiments mentioned above, was parallel to the curves predicted for even mixing. The tidal air for this run was 0.52 litre (calculated from the volumes of air collected in the Douglas bags), the mean effective tidal air derived from the observed mixing curve was 0.203 litre, and the difference between the above figures (mean effective dead space) was 0.317 litre.

The dead space of the model, i.e. the tubes connecting the model to the mixing apparatus (up to and including the box-valve), was calculated from its dimensions to be 0.312 litre. Thus there is close agreement between the measured anatomical dead space and the dead space derived from the mixing curve.

This and other experiments confirmed that where there is no interference with even distribution of the inspired air throughout the F.R.A. the experimental points lie on a line parallel to the predicted curves. In such cases, moreover, an accurate assessment of the anatomical dead space of the model can be made from the mixing curve.

In other studies the even ventilation of the concertina was disturbed by sealing a wide-necked $700 \mathrm{cc}$. bottle inside it (Fig. 5C). This made the model unevenly ventilated since the space within the bottle, with no respiratory excursion, was ventilated only by diffusion. The tidal air ( 0.52 litre) was the same as in Fig. 7 , but it will at once be apparent that the effective tidal air was grossly reduced. Further, the line connecting the experimental points no longer lies parallel to the predicted curves (Fig. 8), but tends to cross them in a manner suggesting that the effective tidal air is progressively diminished as mixing proceeds. Even in the earlier stages of the curve the estimate of dead space (tidal air-effective tidal air) was raised to over 0.40 litre, and it increased to 0.45 litre in the later stages of the mixing curve. The measured external anatomical dead space of the tubes was unchanged at 0.312 litre. Thus uneven ventilation inside the model leads to a considerable increase in estimates of dead space from observation of the mixing curve. The initial, smaller estimated dead space is determined by the rapid nitrogen elimination from the wellventilated space in the model. The subsequent increase in estimated dead space is apparently due to retarded clearing of nitrogen from the poorly ventilated space. This is the only source of nitrogen in the model after the well-ventilated space has been washed out.

\section{Discussion}

Since these studies were completed Bateman (1950) has published his modifications of Cournand's method of studying intrapulmonary mixing. Like Darling et al. (1944) or Cournand et al. (1941) he collected the expired air in a Tissot spirometer. The volume of the channel connecting the mouthpiece to the Tissot spirometer was reduced to a minimum. A fan was used to mix the gases in the spirometer. The rate of nitrogen washout was measured by the analysis of serial samples taken from the spirometer.

The technique described in this study has certain advantages over Bateman's method. The whole mixing study is completed in a single uninterrupted procedure. The apparatus is simpler than that used by Bateman and avoids his arbitrary correction for incomplete mixing in the body of the spirometer. Thus the method, as described in one sample subject and checked by experiments on models, seems to 
be justified for further use in clinical investigation. Modern apparatus, such as a Lilley-Harvey nitrogen meter for gas analysis, would greatly shorten the time required for a single study.

\section{SUMMARY}

An open circuit method of studying the intrapulmonary mixture of gases in which the whole study is completed in a single experiment is described.

The apparent F.R.A. is plotted against the relevant number of breaths, and this is found, by experiments on evenly and unevenly ventilated model lungs, to indicate the presence or absence of poorly ventilated space.

This paper is based on the material for a thesis submitted to the University of tha Witwatersrand for the degree of M.D. My special thanks are due to Professor J. McMichael and to Professor G. Elliott, for their interest in this work, to Dr. J. B. Bateman for a private communication, and to Mr. A. Latham and Mr. C. Lawden, of the Postgraduate Medical School of London, for help with the construction of the apparatus.

\section{REFERENCES}

Bateman, J. B. (1950). J. appl. Physiol., 3, 143.

Bates, D. V., and Christie, R. V. (1950). Clin. Sci., 9, 17.

Birath, G. (1944). Acta med. scand., Suppl. 154.

Briscoe, W. A., Becklake, M. R., and Rose, T. F. (1951). Clin. Sci., 10, 37.

Cournand, A., Baldwin, E. DeF., Darling, R. C., and Richards, D. W. (1941). J. clin. Invest., 20, 681 .

Darling, R. C., Cournand, A., and Richards, D. W. (1944). Ibid., 23, 55.

Gilson, J. C., and Hugh-Jones, P. (1949). Clin. Sci., 7, 185.

Peters, J. P., and Van Slyke, D. D. (1932). Quantitative Clinical Chemistry, vol. 2, p. 218. London.

Robertson, J. S., Siri, W. E., and Jones, H. B. (1950). J. clin. Invest., 29, 577.

\section{A P P E N D I X}

The formula for the prediction curves was derived as follows:

Let $\mathrm{c}=$ functional residual air, $\mathrm{t}=$ effective tidal air, $\mathbf{Z}_{\mathrm{o}}=$ initial concentration of nitrogen in the lungs, $Z_{n}=$ the concentration of nitrogen in the lungs after $n$ breaths, and $\mathrm{C}_{\mathbf{n}}=$ the apparent F.R.A. after $\mathrm{n}$ breaths.

The quantity of nitrogen initially present in the lungs is $Z_{o} c$, and the concentration of nitrogen in the lungs after one inspiration is $Z_{o} \frac{c}{c+t}$. The quantity of nitrogen in the lungs after the second inspiration is $Z_{o} \frac{c}{c+t} c$, and the concentration of nitrogen in the lungs is then $Z_{\circ} \frac{c}{c+t} \cdot \frac{c}{c+t}=Z_{\circ}\left(\frac{c}{c+t}\right)^{2}$.

Thus the concentration of nitrogen in the lungs after $n$ breaths $\left(Z_{n}\right)=Z_{o}\left(\frac{c}{c+t}\right)_{n}$. Let $\frac{c}{c+t}=\beta$, the mixing constant. Then $Z_{n}=Z_{o} \beta^{n}$. The quantity of nitrogen eliminated from the lungs in $n$ breaths is $c\left(Z_{o}-Z_{n}\right)=c\left(Z_{o}-Z_{o} \beta^{n}\right)=c Z_{o}\left(1-\beta^{n}\right)$. The apparent F.R.A. $\left(c_{n}\right)$ which would have eliminated this quantity if completely cleared of nitrogen is $c\left(1-\beta^{n}\right)$. This is the formula used in calculating predicted curves. 